\title{
Theoretical Investigation on the Complexation Characteristics and Spectral Properties of CdTe QDs With Four Capping Agents
}

\section{Qinghong Yang}

Sichuan Normal University

\section{Xujiang Wan}

Sichuan Normal University

\section{Yang Chen}

Sichuan Normal University

Hui Luo

Sichuan Normal University

\section{Yan Zheng}

Sichuan Normal University

Laicai Li ( $D$ lilcmail@163.com )

Sichuan Normal University https://orcid.org/0000-0001-8390-8417

\section{Research Article}

Keywords: CdTe QDs, Capping Agents, Complexation Characteristics, UV-Vis Absorption Spectroscopy, Density Functional Theory

Posted Date: July 8th, 2021

DOl: https://doi.org/10.21203/rs.3.rs-651361/v1

License: (c) (i) This work is licensed under a Creative Commons Attribution 4.0 International License. Read Full License 


\section{Abstract}

In this paper, density functional theory (DFT) and time-dependent density functional theory (TDDFT) are used to study the complexation characteristics CdTe quantum dots with four different capping agents, i.e. : 3-mercaptopropionic acid (MPA), reduced glutathione (GSH), 1-thioglycerol (TG) and 2mercaptoethanesulfonate (MES). The properties of these complexes are analyzed by the complexation energy, bond lengths, electron densities, Mulliken charges and frontier molecular orbitals. In addition, the UV-Vis absorption spectra of pure CdTe QDs and those stable complexes are calculated. The results indicate that the four capping agents could form stable complexes with CdTe QDs. However, there are also some differences. For instance, the complexation between MES and QDs is the most stable and the electron amount transferred from MES to CdTe QDs is the most while the absorption intensity of UVvisible light after complexation is the largest. The stability of the complexes are followed by MPA and TG and the complexation between GSH and QDs is the most unstable, which is accompanied with the minimal electron transfer amount and the weakest absorption intensity of UV-visible light. The maximum absorption wavelengths of CdTe QDs are consistent with the experimental observed wavelength, which explains the experimental phenomena excellently.

\section{Introduction}

In the past two decades, semiconductor nanoparticles have attracted great attention due to their unique optical properties. Quantum dots (QDs) are defined as a semiconductor structure with a physical size smaller than the exciton Bohr radius (the exciton is an electron-hole pair) [1]. When the semiconductor size is less than $100 \mathrm{~nm}$, the structures of these nanomaterials may have tunable characteristics and often exhibit strong quantum confinement effect. This effect leads to excellent size-related optical properties, which has received much interests in different fields such as biomarkers, light emitting diodes and photovoltaic devices [2]. Up till now, quantum dots have been widely used in many fields of life science because of long fluorescence lifetime, excellent biocompatibility and strong anti-photobleaching ability. In addition, the Stokes shift of quantum dots is very large, the excitation spectrum and the emission spectrum can be clearly separated, it is not easy to overlap, and the influence is smaller. [3].

Quantum dots nanocrystals can be synthesized by many methods, such as metal organic synthesis route [4], but this synthesis method requires severe conditions such as high temperature, toxicity and instability. Another method is hydrothermal synthesis route [5]. However, this method may lead to a large number of crystal aggregation and uncontrolled growth to damage the stability of quantum dot nanocrystals and surface defects to damage the optical properties [6]. Therefore, the addition of various surface ligands as capping agents plays an important role as stabilizer in the formation of nanocrystals, which can control the size, shape, growth kinetics, optical properties and surface functionalization of quantum dots [7]. CdTe-thiol systems are one of the most successful cases. The most widely used thiol capping agents are thioglycolic acid (TGA) [8], 3-mercaptopropionic acid (MPA) [9], reduced glutathione (GSH) [10], L-cysteine (L-Cys) [11], sodium 2-mercaptoethanesulfonate (MES) [12], and 1-thioglycerol (TG) [13]. For instance, Zhang et al. used TGA, TG and 2-thioethanolamine as ligands [14] to synthesize stable CdTe 
nanomaterials with one-dimensional structure and controllable aspect ratio. Dong et al. [15] found that the surface of CdTe QDs combined with MPA can remove some trap sites, make the 'dark' quantum dots become 'bright' quantum dots since the fluorescence efficiency of the quantum dots are greatly enhanced. Borchert et al. [16] found that the high photoluminescence quantum yield is attributed to the effective passivation of surface defects by $\mathrm{Cd}$-thiol complexes formed on the surface of nanocrystals. Gong et al. [17] prepared water-soluble CdTe QDs by one-pot method using thioglycolic acid (TGA) as stabilizer and in the presence of L-cysteine and the fluorescence intensity of CdTe QDs increased 1.3 times in comparison with the uncapped structures.

Different capping agents have different binding stability with QDs, and their effects on the optical properties of QDs are also different. Silva et al. [18] studied the complexation stability of GSH, TGA, MPA and TG ligands with CdTe QDs, and found that GSH has higher steric hindrance and weaker complexation, while the other three ligands has stronger complexation with the surface of nanocrystals. Huang et al. [19] studied the effect of three different capping ligands TGA, MPA and GSH on the fluorescence intensity of quantum dots and found that MPA-CdTe has the strongest luminescence, which is followed by GSH-CdTe, while TGA-CdTe has the weakest luminescence, but the differences between the three capping agents are not obvious. Vale et al. [20] found that TGA, MPA, MES and MPS ligands can successfully stablize CdTe QDs, and found that the fluorescence lifetime of CdTe/MES QD is higher than the other three systems. Despite these experimental works, the influences of the capping agents to the properties of quantum dots at atomic level remain to be explored, which need the aid of theoretical calculations. This paper aims to study the complexation properties of four different capping agents MPA, GSH, TG and MES with CdTe QDs by theoretical calculation, and explore the complexation stability and spectral properties of four capping agents on CdTe substrate from the aspects of complexation energies, bond lengths, frontier molecular orbitals and electronic configurations. Based on the obtained results, we hope to reveal the relationship between the complexation stability and spectral characteristics from microscopic point of view.

\section{Calculation Methods}

The molecular structures are optimized using density functional theory (DFT) [21], with the M05 functional implemented in Gaussian09 [22] Inner core electrons of $\mathrm{Cd}$ and Te atoms are treated using pseudopotentials and outer valence electrons are described with LANL2DZ basis sets [23] while $\mathrm{C}, \mathrm{H}, \mathrm{O}, \mathrm{N}$ and $S$ are described with $6-31+G^{*}$ basis sets [24]. Frequency analysises are performed to verify that the optimized structures are indeed minimum energy structures with no imaginary frequencies. For UV-visible absorption spectra, the time-dependent density functional theory (TD-DFT) with the same functional and basis sets [25] is used. Complexation energy is defined as:

$\mathrm{E}_{\text {ads }}=\mathrm{E}_{\text {adsorbate }}+\mathrm{E}_{\text {cluster }}-\mathrm{E}_{\text {adsorbate/cluster }}$

$\mathrm{E}_{\text {absorbate }}$ is the energy of ligand, $\mathrm{E}_{\text {cluster }}$ is the energy of $(\mathrm{CdTe})_{n}$ cluster, $\mathrm{E}_{\text {absorbate/cluster }}$ is the total energy of the complex. The greater the absolute value of $\mathrm{E}_{\mathrm{ads}}$, the higher the complexation energy. 


\section{Results And Discussions}

\section{Complexing energy and bond length analysis of stable complexes}

We chose $(\mathrm{CdTe})_{\mathrm{n}(\mathrm{n}=6,9)}$ clusters as model systems [26] to study the capping effects of 3mercaptopropionic acid (MPA), reduced glutathione (GSH), 1-thioglycerol (TG) and 2mercaptoethanesulfonate (MES) on CdTe QDs. We tried to use different sites such as points, lines and planes on $(\mathrm{CdTe})_{n(n=6,9)}$ to bind different ligands, and obtained a variety of complexes. It is found that when the four ligands are complexed with $(\mathrm{CdTe})_{6}$, only one $\mathrm{Cd}$ atom on the surface of the six-membered ring and two $\mathrm{Cd}$ atoms on the surface are simultaneously complexed to form a stable complexes. When complexing with (CdTe $)_{9}$, in addition to the formation of the same stable complexes as (CdTe $)_{6}$, it also combines with $\mathrm{Cd}$ atoms at the bridge site of $(\mathrm{CdTe})_{9}$ to form a stable complexes. The optimized stable complexes are listed in Fig. 1 and Fig. S1. Complexation energies and some crucial bond lengths are listed in Table 1. In the diagram, A-MPA, A-GSH, A-TG and A-MES represent stable complexes using 3mercaptopropionic acid, reduced glutathione, 1-thioglycerol and 2-mercaptoethanesulfonate as capping agents and the numbers represent different binding sites $(\mathrm{CdTe})_{n(n=6,9)}$, respectively.

Table 1

The complexation energy and bond length of MPA口GSH口TG and MES on $(\mathrm{CdTe})_{n(n=6,9)}$ respectively

\begin{tabular}{|llllll|}
\hline complexes & $\begin{array}{l}\text { Energy } \\
\text { kcal/mol }\end{array}$ & $\begin{array}{l}\text { bond length } \mathbf{r} / \\
\text { complexes }\end{array}$ & $\begin{array}{l}\text { energy } \\
\text { kcal/mol }\end{array}$ & bond length $\mathbf{r} / \AA$ \\
\hline A1-MPA & 49.88 & & A1-GSH & 34.12 & \\
\hline A2-MPA & 57.73 & 2.524 & A2-GSH & 46.66 & 2.560 \\
\hline A3-MPA & 65.20 & & A3-GSH & 48.40 & \\
\hline A4-MPA & 67.38 & 2.496 & A4-GSH & 59.01 & 2.547 \\
\hline A5-MPA & 64.50 & 2.514 & A5-GSH & 54.17 & 2.522 \\
\hline A1-TG & 55.08 & & A1-MES & 82.87 & \\
\hline A2-TG & 56.22 & 2.520 & A2-MES & 84.29 & 2.461 \\
\hline A3-TG & 71.14 & & A3-MES & 103.95 & \\
\hline A4-TG & 67.11 & 2.496 & A4-MES & 112.68 & 2.446 \\
\hline A5-TG & 67.15 & 2.492 & A5-MES & 90.11 & 2.460 \\
\hline
\end{tabular}

Among the complexes formed by using MPA as capping agent, A2-MPA is the most stable complexes on $(\mathrm{CdTe})_{6}$, with the complexation energy of $57.73 \mathrm{kcal} / \mathrm{mol}$ and bond length of the formed Cd1-S bond is 
$2.524 \AA$. In the same time, A4-MPA is the most stable complexes on $(\mathrm{CdTe})_{9}$, with the complexation energy of $67.38 \mathrm{kcal} / \mathrm{mol}$ and bond length of the formed Cd1-S bond is $2.496 \AA$. When GSH interacts with $(\mathrm{CdTe})_{6}$ and (CdTe) ${ }_{9}$, respectively. A2-GSH is the most stable complexes on (CdTe) ${ }_{6}$ and A5-GSH is the most stable complexes on (CdTe) ${ }_{9}$. Their complexation energies are $54.60 \mathrm{kcal} / \mathrm{mol}$ and $59.69 \mathrm{kcal} / \mathrm{mol}$, respectively. The bond lengths of the formed Cd1-S bonds are $2.570 \AA \AA$ and $2.533 \AA$. For capping agent TG, A2-TG and A3-TG are the most stable complexes on (CdTe $)_{6}$ and $(\mathrm{CdTe})_{9}$, respectively. The complexation energy of A3-TG is higher than A2-TG. The complexation energy of A3-TG is $71.14 \mathrm{kcal} / \mathrm{mol}$ while A2-TG is $56.22 \mathrm{kcal} / \mathrm{mol}$. In addition, the bond lengths of the formed Cd1-S bonds is $2.520 \AA$. As the last capping agent MES, A2-MES is the most stable complexes on (CdTe $)_{6}$. It's complexation energy and bond length of Cd1-S are $84.29 \mathrm{kcal} / \mathrm{mol}$ and $2.461 \AA$, respectively. When MES interacts with (CdTe) $)_{9}$. A4MES is the most stable complexes, with the complexation energy of $112.68 \mathrm{kcal} / \mathrm{mol}$ and bond length of Cd1-S 2.446 $\AA$. Based on these result, we can find that the complexes formed using capping agents MPA, $\mathrm{GSH}, \mathrm{TG}$ and MES with (CdTe) ${ }_{9}$ is more stable than that those formed by (CdTe) ${ }_{6}$. Table 1 shows that the complexation energies using capping agents MES on $(\mathrm{CdTe})_{\mathrm{n}(\mathrm{n}=6,9)}$ are significantly greater than other three capping agents at the corresponding site of the substrate, and the bond lengths are also shorter than that of the other three capping agents, indicating that the complexes using the capping agent MES on $(\mathrm{CdTe})_{n(n=6,9)}$ are relatively stable. For the other three capping agents, the complexation energies of GSH with (CdTe $)_{n(n=6,9)}$ QDs is significantly smaller than that of MPA and TG, indicating that the complexation of GSH with $(\mathrm{CdTe})_{n(n=6,9)}$ QDs are the most unstable complexes, which is consistent with the experimental results of silva et al. [18].

\section{Energy level analysis of the stable complexes}

In order to compare the complexation abilities of these four ligands on $(\mathrm{CdTe})_{n(n=6,9)}$ QDs, we calculated the frontier molecular orbital energies of $(\mathrm{CdTe})_{n(n=6,9)}$ QDs and ligands before complexing, as shown in Fig. 2. The frontier molecular orbitals energies of stable complexes are shown in Table 2. 
Table 2

$\mathrm{HOMO}(\mathrm{eV}) \square \mathrm{LUMO}(\mathrm{eV})$ and $\triangle \mathrm{Eq}(\mathrm{eV})$ of $(\mathrm{CdTe})_{\mathrm{n}(\mathrm{n}=6,9)}$ before adsorption and stable structures after adsorption

\begin{tabular}{|llllllll|}
\hline complexes & HOMO & LUMO & $\Delta \mathrm{E}_{\mathrm{q}}$ & complexes & HOMO & LUMO & $\Delta \mathrm{E}_{\mathrm{q}}$ \\
\hline 6*6 & 6.28 & 2.49 & 3.79 & $9 * 9$ & 6.07 & 2.58 & 3.49 \\
\hline A1-MPA & 2.73 & -0.29 & 3.02 & A1-GSH & 2.98 & -0.03 & 3.01 \\
\hline A2-MPA & 3.06 & -0.25 & 3.31 & A2-GSH & 3.50 & -0.20 & 3.70 \\
\hline A3-MPA & 2.96 & 0.22 & 2.74 & A3-GSH & 3.47 & 0.28 & 3.19 \\
\hline A4-MPA & 3.45 & 0.14 & 3.31 & A4-GSH & 3.61 & 0.14 & 3.47 \\
\hline A5-MPA & 3.41 & 0.17 & 3.24 & A5-GSH & 3.76 & 0.47 & 3.29 \\
\hline A1-TG & 2.58 & -0.59 & 3.17 & A1-MES & 0.99 & -1.78 & 2.77 \\
\hline A2-TG & 3.06 & -0.21 & 3.27 & A2-MES & 1.04 & -2.02 & 3.06 \\
\hline A3-TG & 3.24 & 0.20 & 3.04 & A3-MES & 1.47 & -1.40 & 2.87 \\
\hline A4-TG & 3.52 & 0.23 & 3.29 & A4-MES & 1.21 & -1.31 & 2.52 \\
\hline A5-TG & 3.42 & 0.30 & 3.12 & A5-MES & 1.03 & -1.35 & 2.38 \\
\hline
\end{tabular}

It can be seen from Fig. 2 that before the complexation, the HOMO and LUMO values of (CdTe) ${ }_{6}$ QDs are -6.28 and $-2.49 \mathrm{eV}$, respectively. The HOMO and LUMO values of (CdTe) ${ }_{9} \mathrm{QDs}$ are $-6.07 \mathrm{eV}$ and -2.58 $\mathrm{eV}$, respectively. The HOMO and LUMO values of the capping agent MPA are $-0.14 \mathrm{eV}$ and $2.64 \mathrm{eV}$, respectively. The HOMO and LUMO values of the capping agent GSH are $-1.31 \mathrm{eV}$ and $1.44 \mathrm{eV}$, respectively. The HOMO and LUMO values of the capping agent TG are $-0.25 \mathrm{eV}$ and $3.16 \mathrm{eV}$, respectively. The HOMO and LUMO values of the capping agent MES are $2.61 \mathrm{eV}$ and $6.97 \mathrm{eV}$, respectively. From the above data, we can see that the HOMO energies of the four capping agents are higher than the LUMO energies of the both $(\mathrm{CdTe})_{6}$ and $(\mathrm{CdTe})_{9} \mathrm{QDs}$, thus the charge transfer from the four capping agent to $(\mathrm{CdTe})_{n(n=6,9)}$ should be quite easy, indicating that they can be used as excellent ligands for CdTe QDs. In comparison, the HOMO energy level of the capping agent MES is higher than the other three capping agents, and it is easiest to transfer electrons to CdTe QDs. The HOMO energy level of the capping agent GSH is the lowest, indicating that the electron transfer of the capping agent GSH to CdTe QDs is the weakest, and the stability of GSH-(CdTe $)_{n(n=6,9)}$ is therefore relatively weaker.

It can be seen from Table 2 that the HOMO and LUMO energy levels of the stable complexes are greatly reduced, and the energy gap value $(\triangle \mathrm{Eq})$ is also significantly reduced. The $\triangle \mathrm{Eq}$ of $(\mathrm{CdTe})_{6}$ decreases from $3.79 \mathrm{eV}$ before the complexing to $3.02 \mathrm{eV}$ and $3.31 \mathrm{eV}$ after complexation MAP, and (CdTe) decreases from $3.49 \mathrm{eV}$ to $2.74 \mathrm{eV}, 3.31 \mathrm{eV}$ and $3.24 \mathrm{eV}$. The $\triangle \mathrm{Eq}$ of $(\mathrm{CdTe})_{6}$ decreases from $3.79 \mathrm{eV}$ before complexing to $3.01 \mathrm{eV}$ and $3.70 \mathrm{eV}$ after complexing $\mathrm{GSH}$, and (CdTe), decreases from $3.49 \mathrm{eV}$ to $3.19 \mathrm{eV}, 3.47 \mathrm{eV}$ and $3.29 \mathrm{eV}$. The $\triangle \mathrm{Eq}$ of $(\mathrm{CdTe})_{6}$ decreases from $3.79 \mathrm{eV}$ before complexing to $3.17 \mathrm{eV}$ 
and $3.27 \mathrm{eV}$ after complexing TG, and (CdTe) ${ }_{9}$ decreases from $3.49 \mathrm{eV}$ to $3.04 \mathrm{eV}, 3.29 \mathrm{eV}$ and $3.12 \mathrm{eV}$. The $\triangle \mathrm{Eq}$ of (CdTe $)_{6}$ decreases from $3.79 \mathrm{eV}$ before complexation to $2.77 \mathrm{eV}$ and $3.06 \mathrm{eV}$ after complexating MES, and (CdTe) ${ }_{9}$ decreases from $3.49 \mathrm{eV}$ to $2.87 \mathrm{eV}, 2.52 \mathrm{eV}$ and $2.38 \mathrm{eV}$. Comparing the calculation results of four kinds of capping agents with CdTe QDs, the HOMO level and LUMO level of the stable configuration formed by the capping agent MES complex on $(\mathrm{CdTe})_{n(n=6,9)}$ are reduced more, and the $\triangle E q$ is also reduced more, indicating that the capping agent MES can produce more dispersed electron transferring to CdTe QDs to form a more stable complex, and $\triangle \mathrm{Eq}$ of the complex is smaller, which is beneficial to the electron excitation inside the complex, so it is easier to absorb light energy and produce molecular fluorescence. The HOMO energy level and LUMO energy level of the stable configuration formed by the complexation of GSH with $(\mathrm{CdTe})_{n(n=6,9)}$ decreases the least, and the $\triangle \mathrm{Eq}$ also decreases the least, indicating that the electron transfer between GSH and $(\mathrm{CdTe})_{n(n=6,9)}$ is the weakest among the four kinds of capping agents, and the complexation is also the most unstable, which is consistent with the previous analysis.

\section{Frontier molecular orbital and electronic structure analysis of stable complexes}

Figure 3 depicts the frontier molecular orbitals of $(\mathrm{CdTe})_{n(n=6,9)}, M P A, G S H, T G$ and MES before complexation, including the highest occupied molecular orbital ( $\mathrm{HOMO}$ ) and the lowest unoccupied molecular orbital (LUMO). It can be seen that the HOMO orbital of $(\mathrm{CdTe})_{n(n=6,9)}$ before the complexation is mainly localized on the Te atoms, and the LUMO orbital are mainly localized on the $\mathrm{Cd}$ atoms, indicating that $\mathrm{Te}$ atom possesses the ability to provide electrons and the $\mathrm{Cd}$ atom possesses the ability to accept electrons in $(\mathrm{CdTe})_{n(n=6,9)}$. The HOMO orbitals of MPA, GSH, TG and MES molecules are mainly localized on the $S$ atoms which are located on the end of the molecules and the LUMO orbitals are mainly located on the other end. Such results indicate that the ability to provide electrons in these four capping agents are dependent on the $S$ atom. Due to the energy of HOMO orbital of $S$ atom in capping agents is higher than that of LUMO of Cd atom in $(\mathrm{CdTe})_{n(n=6,9)}$. The $S$ atom with charge-donating properties in the capping agents MPA, GSH, TG and MES molecules is easy to transfer electrons to $\mathrm{Cd}$ atom with electronaccepting ability in $(\mathrm{CdTe})_{n(n=6,9)}$. The complexation process is realized by transferring the lone pair electrons of $\mathrm{S}$ atom to the empty orbital of $\mathrm{Cd}$ atom. From the complexation configuration diagram in Fig. 1, we can also see that all the complexation mode of the capping agents MPA, GSH, TG and MES with $(\mathrm{CdTe})_{n(n=6,9)}$ can be attributed to the interactions between the $S$ atom of the capping agents and the $\mathrm{Cd}$ atom of the quantum dots, and the complexation energies are very large and thus the complexes are stable.

Figure $\mathrm{S} 2$ shows the electron density maps of the stable configurations of the four capping agents MPA, GSH, TG and MES on the substrate $(\mathrm{CdTe})_{n(n=6,9)}$, which is useful to determine whether the capping agents are complexed with (CdTe $)_{6}$ and $(\mathrm{CdTe})_{9}$. It can be seen from Figure S2, four kinds of capping agents and CdTe QDs have obvious overlap of electron cloud, indicating that there are electronic interactions between the CdTe QDs and the capping agents. 
In order to quantitatively analyze the electronic interaction between the four capping agents MPA, GSH, TG and MES and the substrate $(\mathrm{CdTe})_{n(n=6,9)}$, we have also listed the Mulliken charge values of $\mathrm{Cd}$ and Te atoms on (CdTe $)_{n(n=6,9)}$ and $S$ atom of the four capping agents before the complexation and the corresponding atoms in the stable configurations after the complexation, which are listed in Table S1. It can be seen from the results that among all the corresponding configurations, the charge transferred from the capping agent MES to CdTe QDs is the most, followed by MPA and TG, and the least is GSH. This is consistent with the stability order of the complexes formed by MPA, GSH, TG and MES with CdTe quantum dots. The coordination of functional groups in organic molecules with metal ions on the surface of quantum dots will change the surface state of quantum dots, affect the luminescence process, and lead to changes in the fluorescence lifetime of quantum dots [27]. The influence of different organic ligands on the fluorescence properties of quantum dots is mainly related to the electron-donating or electron-absorbing properties of coordination groups [28]. When the surface ligands with electrondonating groups are coordinated with the surface of quantum dots, the surface defects of quantum dots can be effectively removed, resulting in fluorescence enhancement [29]. The above analysis of charge transfer ability may explain the phenomenon that the fluorescence intensity of MPA-capped QDs reported in the experiment is slightly larger than that of GSH-capped QDs [19].

\subsection{Analysis of UV-Vis absorption spectra of coordination configuration}

It is pointed out that the change of fluorescence signal caused by the surface effect of quantum dots is due to the formation of ground state complexes between the organic functional groups on the surface of quantum dots and metal ions, which changes the surface state of quantum dots and tends to cause significant changes in the UV-Vis absorption intensity [30]. Therefore, we calculated the UV-Vis absorption spectra of CdTe QDs, as shown in Fig. 4(a); And the UV-Vis absorption spectra of stable complexes formed between capping agents MPA, GSH, TG and MES and (CdTe $)_{n(n=6,9)}$ are shown in Fig. 4(b), 4(c), 4(d) and 4(e). It can be seen from Fig. 4(a) that the maximum absorption wavelengths of (CdTe) ${ }_{6}$ and $(\mathrm{CdTe})_{9}$ are located around $400 \mathrm{~nm}$. The maximum absorption wavelength range of CdTe QDs obtained in the experiment is 400-500 nm [31-32], which is in good agreement with our calculation results. Comparing Figs. 4(a), 4(b), 4(c), 4(d) and 4(e), it can be seen that the UV-visible absorption intensity of CdTe QDs is significantly enhanced after the addition of capping agents MPA, GSH, TG and MES. According to the analysis by Zhu et al. [29], MPA, GSH, TG and MES have strong coordination with QDs, which changes the surface state of QDs and causes the change of UV-Vis absorption intensity of CdTe QDs. However, compared with Fig. 4(b), 4(c), 4(d) and 4(e), it can be seen that the UV-visible absorption intensity of the QDs coordinated by MES is the largest, followed by MPA and TG, and GSH is the smallest. It indicates that among the four ligands, the coordination between the capping agent MES and CdTe QDs is the strongest, followed by MPA and TG, and GSH is the weakest. This is consistent with the previous analysis.

\section{Conclusions}


In this paper, the density functional theory and time-dependent density functional theory are used to study the complex characteristics between the capping agents MPA, GSH, TG, MES and CdTe QDs, respectively. The results indicate that MPA, GSH, TG and MES have strong interaction with CdTe QDs through the analysis of complexation energies, bond lengths, electron densities, Mulliken charges and frontier molecular orbitals. Among them, MES-CdTe complexes are the most stable complexes after complexation, which is also accompanied with the largest electrons transfers from MES to CdTe QDs, the strongest interaction energy between MES and CdTe QDs. The complexation energies are followed by MPA and TG while GSH is the weakest when forming complexes. In addition, the UV-Vis absorption spectra of each stable complexes are calculated. The maximum absorption wavelength of CdTe QDs is within the wavelength range measured in the experiment, indicating that the models and theoretical methods are reliable. The UV-Vis absorption intensity of MES-coordinated QDs is the strongest, which is followed by MPA and TG while GSH is the weakest. This indicates that the absorption intensity is closely related with the complexation energies between four capping agents and CdTe QDs, which might finally have some influences on their fluorescence Our research can provide theoretical support for the experimental use of MPA, GSH, TG and MES as capping agents for CdTe QDs.

\section{Declarations}

\section{Acknowledgements}

This project is supported by High Performance Computing Centre of Sichuan Normal University, China.

Funding: N/A

Conflicts of interest/Competing interests: The authors declare no conflicts of interest.

Availability of data and material: The data can be accessed from the author directly upon reasonable requests.

Code availability: N/A

Authors' contributions: Qinghong Yang, Data curation, formal analysis, and writing-original draft; Xujiang Wan, data curation, writing and reviewing; Yang Chen, data curation; Hui Luo, data curation; Yan Zheng, supervision. Laicai Li, supervision and editing.

\section{References}

1. Alivisatos AP (1996) Semiconductor Clusters, Nanocrystals, and Quantum Dots. Science 271:933937

2. Park J, Joo J, Kwon SG, Jang YJ, Hyeon T (2007) Synthesis of Monodisperse Spherical Nanocrystals. Angew. Chem. Int. Ed. 46:4630-4660 
3. Shinchi H, Wakao M, Nagata N, Sakamoto M, Mochizuki E, Uematsu T, Kuwabata S, Suda Y (2014) Cadmium-free sugar-chain-immobilized fluorescent nanoparticles containing low-toxicity ZnS-AgInS 2 cores for probing lectin and cells. J. Am. Chem. Soc. 25:286-295

4. Peng ZA, Peng X (2001) Formation of high-quality CdTe, CdSe, and CdS nanocrystals using CdO as precursor. J. Am. Chem. Soc. 123:183-184

5. Sheng ZH, Han HY, Hu XF, Chen C (2010) One-step growth of high luminescence CdTe quantum dots with low cytotoxicity in ambient atmospheric conditions. Dalton Trans. 39:7017-7020

6. Rogach AL, FranzI T, Klar TA, Feldmann J, Gaponik N, Lesnyak V, Shavel A, Eychmüller A, Rakovich YP, Donegan JF (2007) Aqueous synthesis of thiol-capped CdTe nanocrystals: state-of-the-art. J. Phys. Chem. C 111:14628-14637

7. Green M (2010) The nature of quantum dot capping ligands. J. Mater. Chem. 20:5797-5809

8. Rodrigues SS, Prieto DR, Ribeiro DS, Barrado E, Prior JA, Santos JL (2015) Competitive metal-ligand binding between CdTe quantum dots and EDTA for free $\mathrm{Ca}^{2+}$ determination. Talanta 134:173-182

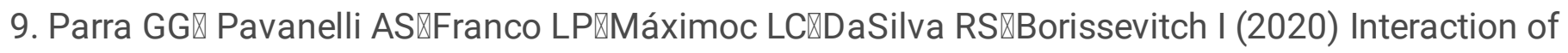
CdTe-MPA quantum dots with meso-tetra methyl pyridyl porphyrin. Charge transfer complex formation. J. Photochem. Photobiol., A 398:112580

10. Tan XP, Li Q, Yang JD (2020) A simple fluorescence method detection levofloxacin in milk based on GSH-CdTe QDs. J. Mol. Struct. 1201:127175

11. Razavi M, Kompany-Zareh M, Khoshkam M (2021) PARAFAC study of L-cys@CdTe QDs interaction to BSA, cytochrome $c$ and trypsin: An approach through electrostatic and covalent bonds. Spectrochim. Acta, Part A 246:119016

12. Paim AP, Rodrigues SM, Ribeiro DM, de Souza GS, Santos JM, Araújo AN, Amorim CG, Teixeira-Neto É, da Silva VL, Montenegro MM (2017) Fluorescence probe for mercury(II) based on the aqueous synthesis of CdTe quantum dots stabilized with 2-mercaptoetanosulfonate. New J. Chem. 41:32653272

13. Luo XT, Han JS, Ning Y, Lin Z, Zhang H, Yang B (2011) Polyurethane-based bulk nanocomposites from 1-thioglycerol-stabilized CdTe quantum dots with enhanced luminescence. J. Mater. Chem. 21:6569-6575

14. Zhang H, Wang DY, Yang B, Möhwald H (2006) Manipulation of Aqueous Growth of CdTe Nanocrystals To Fabricate Colloidally Stable One-Dimensional Nanostructures. J. Am. Chem. Soc. 128:10171-10180

15. Dong CQ, Qian HF, Fang NH, Ren JC (2006) Study of Fluorescence Quenching and Dialysis Process of CdTe Quantum Dots, Using Ensemble Techniques and Fluorescence Correlation Spectroscopy. J. Phys. Chem. B 110:11069-11075

16. Borchert H, Talapin DV, Gaponik N, McGinley C, Adam S, Lobo A, Möller T, Weller H (2003) Relations between the Photoluminescence Efficiency of CdTe Nanocrystals and Their Surface Properties Revealed by Synchrotron XPS. J. Phys. Chem. B 107:9662-9668 
17. Gong TT, Liu JF, Liu XX, Liu J, Xiang JK, Wu YW (2016) A sensitive and selective sensing platform based on CdTe QDs in the presence of L-cysteine for detection of silver, mercury and copper ions in water and various drinks. Food Chem. 213:306-312

18. Silva FO, Carvalho MS, Mendonça R, Macedo WA, Balzuweit K, Reiss P, Schiavon MA (2012) Effect of surface ligands on the optical properties of aqueous soluble CdTe quantum dots. Nanoscale Res. Lett. 7:536-539

19. Huang K, Xu KL, Tang J, Yang L, Zhou JR, Hou XD, Zheng CB (2015) Room Temperature Cation Exchange Reaction in Nanocrystals for Ultrasensitive Speciation Analysis of Silver lons and Silver Nanoparticles. Anal. Chem. 87:6584-6591

20. Vale BC, Mourão RS, Bettini J, Sousa JL, Ferrari JL, Reiss P, Aldakov D, Schiavon MA (2019) Ligand induced switching of the band alignment in aqueous synthesized CdTe/CdS core/shell nanocrystals. Sci. Rep. 9:8332

21. Miehlich B, Savin A, Stoll H, Preuss H (1989) Results obtained with the correlation energy density functionals of Becke and Lee,Yang and Parr. Chem. Phys. Lett. 157:200-206

22. Karunakaran R, Cherumuttathu HS (2013) Which density functional is close to CCSD accuracy to describe geometry and interaction energy of small noncovalent dimers? A benchmark study using Gaussian09.J. Comput. Chem. 34:1341-1353

23. Legge FS, Nyberg GL, Peel JB (2001) DFT Calculations for Cu-, Ag-, and Au-Containing Molecules. J. Phys. Chem. A 105:7905-7916

24. Domingo LR, Sáez JA, Joule JA, Rhyman L, Ramasami P (2013) A DFT Study of the [3+2] versus [4+2] Cycloaddition Reactions of 1,5,6-Trimethylpyrazinium-3-olate with Methyl Methacrylate. J. Org. Chem. 78:1621-1629

25. Hu CP, Sugino O, Miyamoto Y (2006) Modified linear response for time-dependent density-functional theory: Application to Rydberg and charge-transfer excitations. Phys. Rev. A 74:032508

26. Hu PY, Zhou RH, Wang DY, Luo H, Xiong XL, Huang K, Li LC, Chen PP (2020) Cysteine mediated synthesis of quantum dots: Mechanism and application in visual detection of hydrogen peroxide and glucose. Sens. Actuators, B 308:127702

27. Peterson MD, Cass LC, Harris RD, Edme K, Sung K, Weiss EA (2014) The role of ligands in determining the exciton relaxation dynamics in semiconductor quantum dots. Annu. Rev. Phys. Chem. 65:317339

28. Dennis AM, Sotto DC, Mei BC, Medintz IL, Mattoussi H, Bao G (2010) Surface ligand effects on metalaffinity coordination to quantum dots: implications for nanoprobe self-assembly. Bioconjugate Chem. 21:1160-1170

29. Zhu WF, Wang J, Xie SQ, Zhu YQ, Wang LM, Xu JJ, Liao S, Ren JW, Liu Q, Yang H, Chen XQ (2020) LPyroglutamic Acid-Modified CdSe/ZnS Quantum Dots: A New Fluorescence-Responsive Chiral Sensing Platform for Stereospecific Molecular Recognition. Anal. Chem. 92:12040-12048

30. Knauf RR, Lennox JC, Dempsey JL (2016) Quantifying Ligand Exchange Reactions at CdSe Nanocrystal Surfaces. Chem. Mater. 28:4762-4770 
31. Chen C, He XW, Gao L, Ma N (2013) Cation Exchange-Based Facile Aqueous Synthesis of Small, Stable, and Nontoxic Near-Infrared $\mathrm{Ag}_{2} \mathrm{Te} / \mathrm{ZnS}$ Core/Shell Quantum Dots Emitting in the Second Biological Window. ACS Appl. Mater. Interfaces 5:1149-1155

32. Smith AM, Niu SM (2011) Bright and Compact Alloyed Quantum Dots with Broadly Tunable NearInfrared Absorption and Fluorescence Spectra through Mercury Cation Exchange. J. Am. Chem. Soc. 133:24-26

\section{Figures}
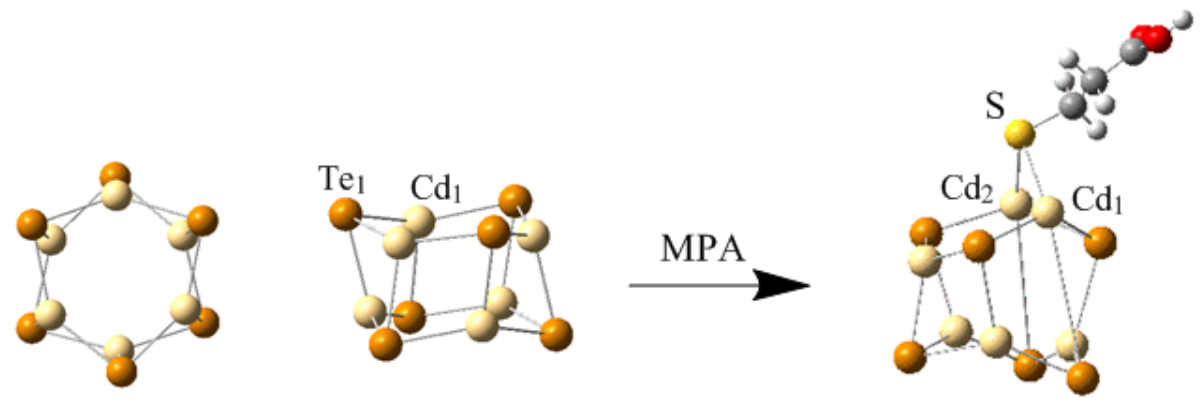

A1-MPA
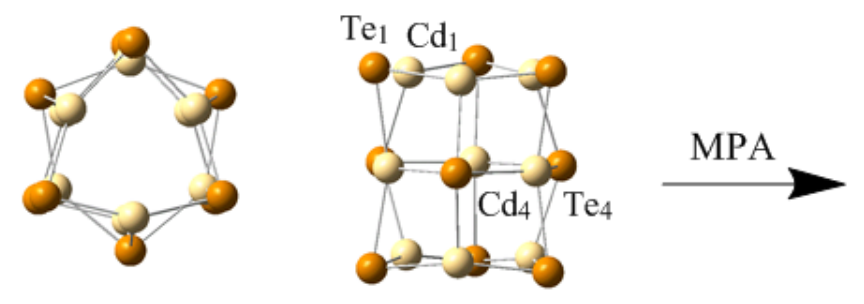

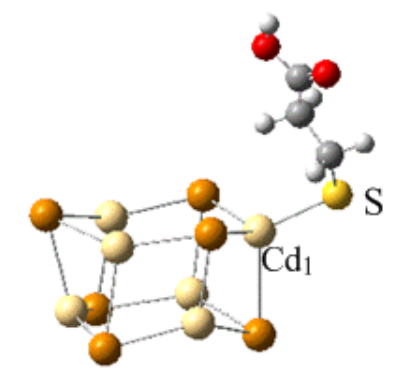

A2-MPA

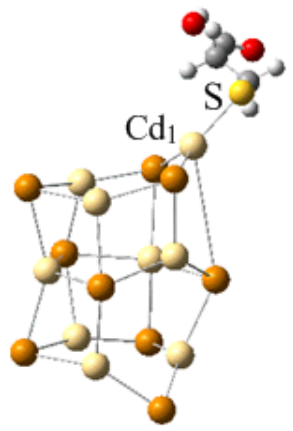

A4-MPA

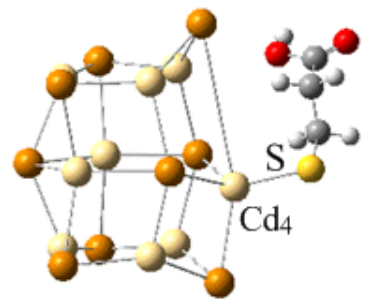

A5-MPA

Figure 1

The stable complexes of MPA on $(\mathrm{CdTe}) n(n=6,9)$ respectively 


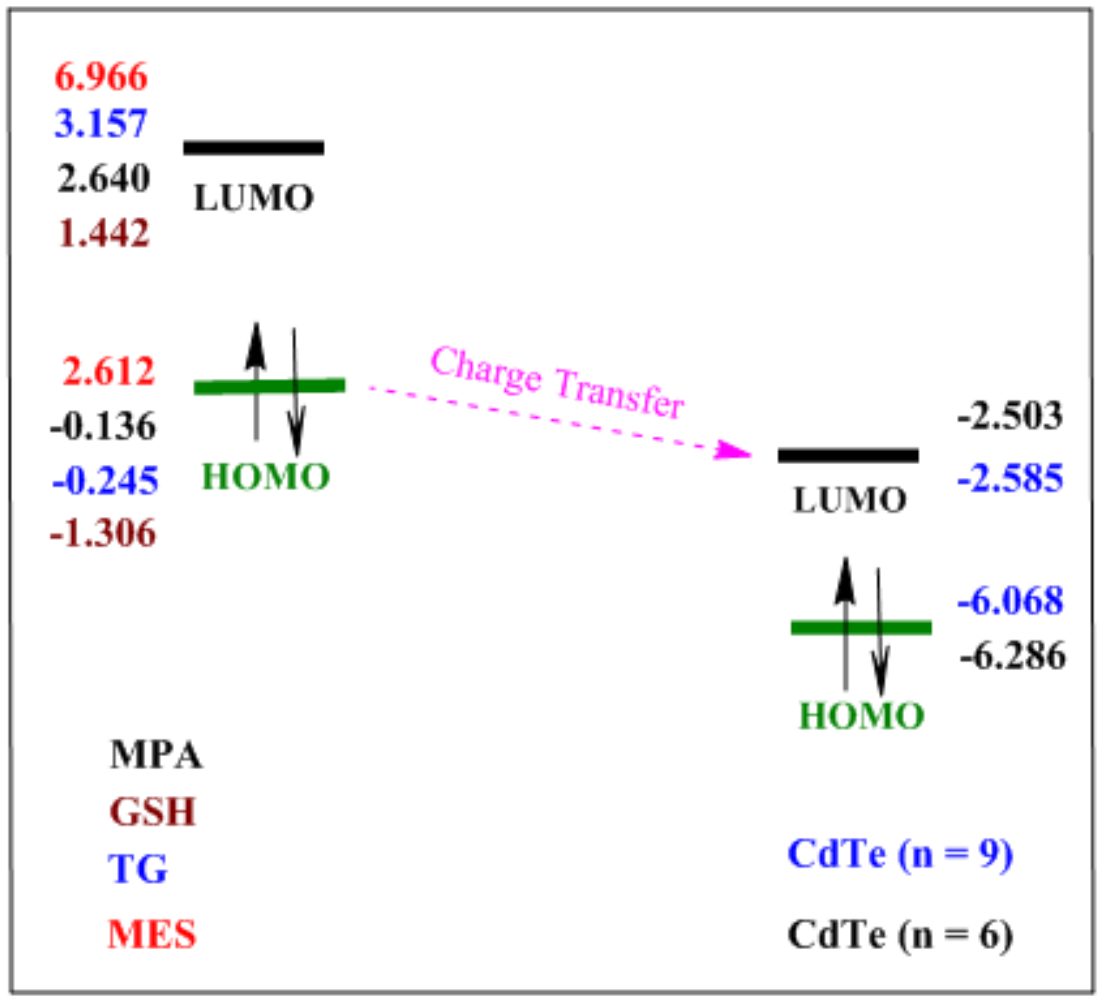

Figure 2

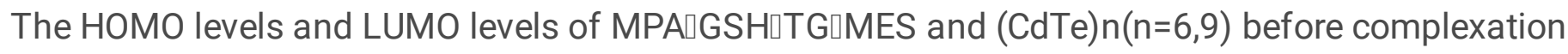
Configuration HOMO LUMO
Configuration
НОМо
LUMO

$(\mathrm{CdTe})_{6}$
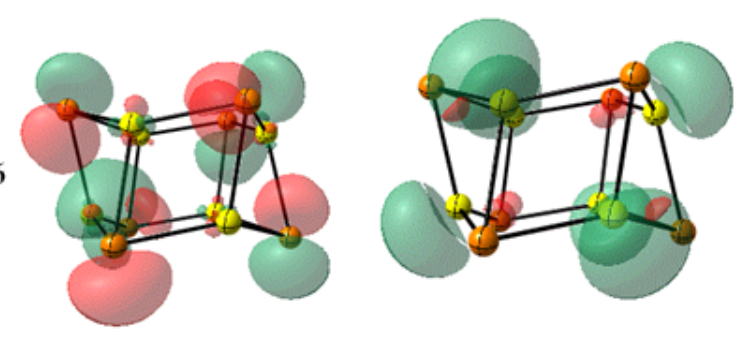

$(\mathrm{CdTe})_{9}$
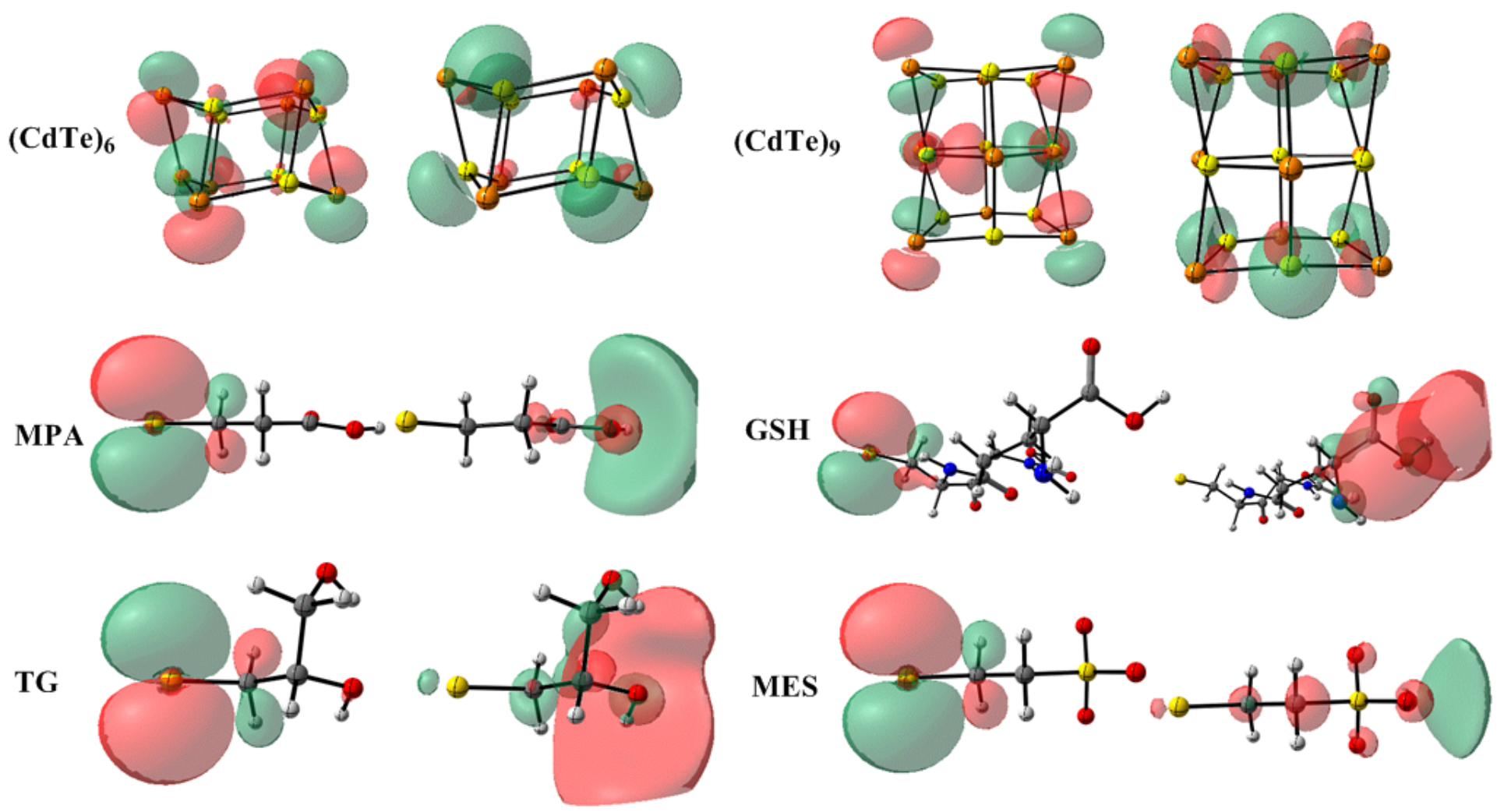

Figure 3 
The frontier molecular orbitals diagram of MPAIGSHITG and MES before and after complexation on $(\mathrm{CdTe}) \mathrm{n}(\mathrm{n}=6,9)$
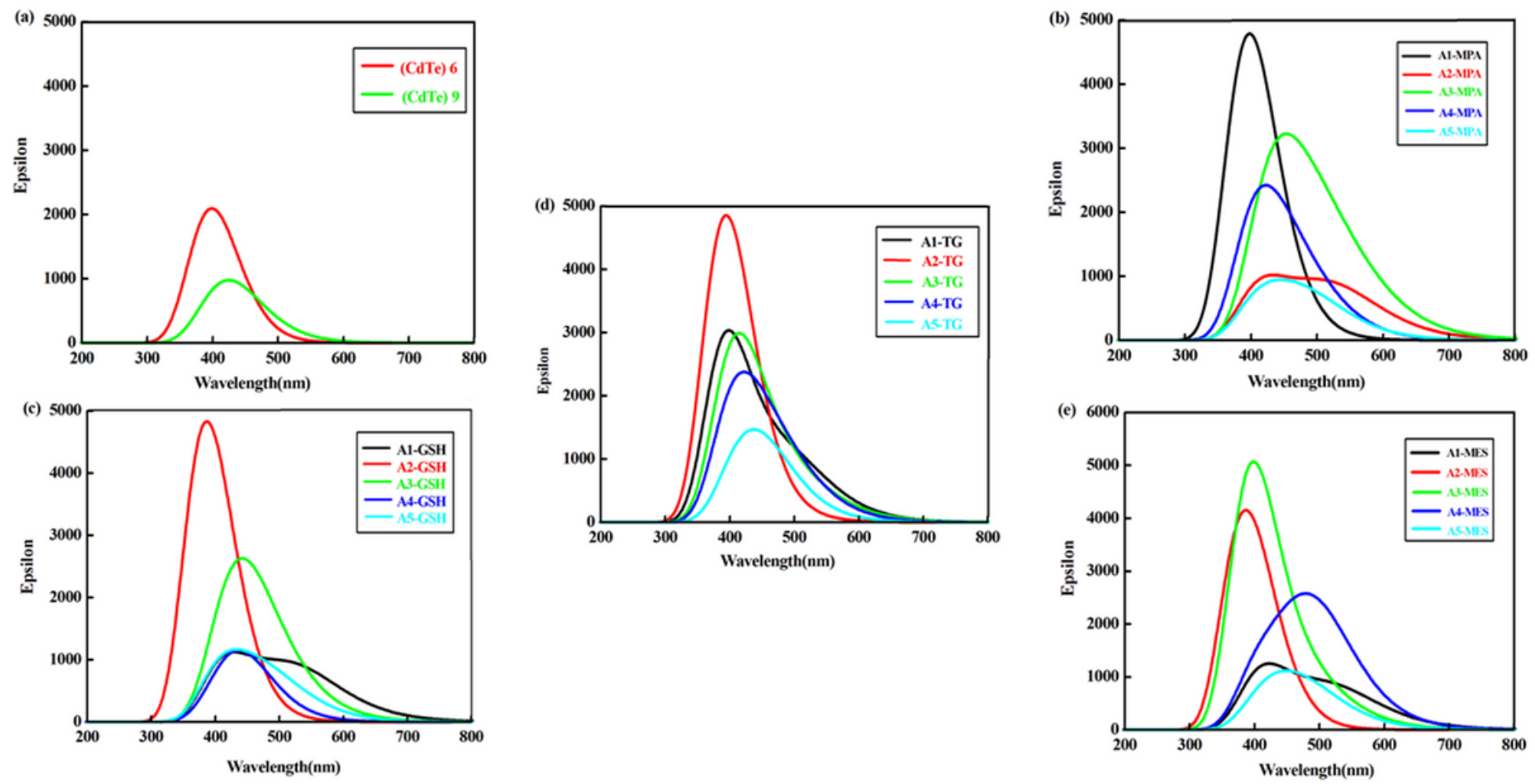

Figure 4

UV-Vis absorption spectrum of bare CdTe QDs (a) and stable complexes of MPA(b) $\square G S H(c) \square T G(d)$ and MES(e) after complexation

\section{Supplementary Files}

This is a list of supplementary files associated with this preprint. Click to download.

- GraphicalAbstract.doc

- Supportinginformation.docx 